\title{
Solar and Heliospheric Physics
}

\section{Silvia Dalla*}

Jeremiah Horrocks Institute, University of Central Lancashire, Preston, PR1 2HE, UK

E-mail: sdalladuclan.ac.uk

This rapporteur report summarises contributions to the SH sessions at the 36th ICRC. Topics include energetic particles accelerated at/near the Sun and in interplanetary space, anomalous cosmic rays and galactic cosmic rays within the heliosphere. A review is presented of the main new findings in observational, theoretical and computational work on solar and heliospheric particle populations, their acceleration, transport and effects on the near-Earth environment. Finally future missions and new proposed instrumentation are discussed.

36th International Cosmic Ray Conference -ICRC2019-

July 24th - August 1st, 2019

Madison, WI, U.S.A.

${ }^{*}$ Speaker. 


\section{Introduction}

Contributions on Solar and Heliospheric Physics at the 36th International Cosmic Ray Conference consisted of 52 talks and 120 posters, covering a broad range of topics and considering many heliospheric environments including the Sun, the interplanetary medium and the outer heliosphere.

In this review, contributions are organised according to three main themes:

- Where and how is particle acceleration taking place?

- How is propagation processing particle fluxes, spectra and other observables?

- How have conditions in the heliosphere evolved over time?

In addition to the papers mentioned in this rapporteur review, a number of other highly interesting and thorough studies were also presented at the conference, and are included within these Proceedings. It was not possible to cover all contributions in this paper, in which only a subset of topics will be reported on.

\section{Where and how is particle acceleration taking place?}

\subsection{Solar Energetic Particles}

Solar Energetic Particles (SEPs), accelerated during Coronal Mass Ejections (CMEs) and flares, have been routinely detected by spacecraft instrumentation for decades. The energy range

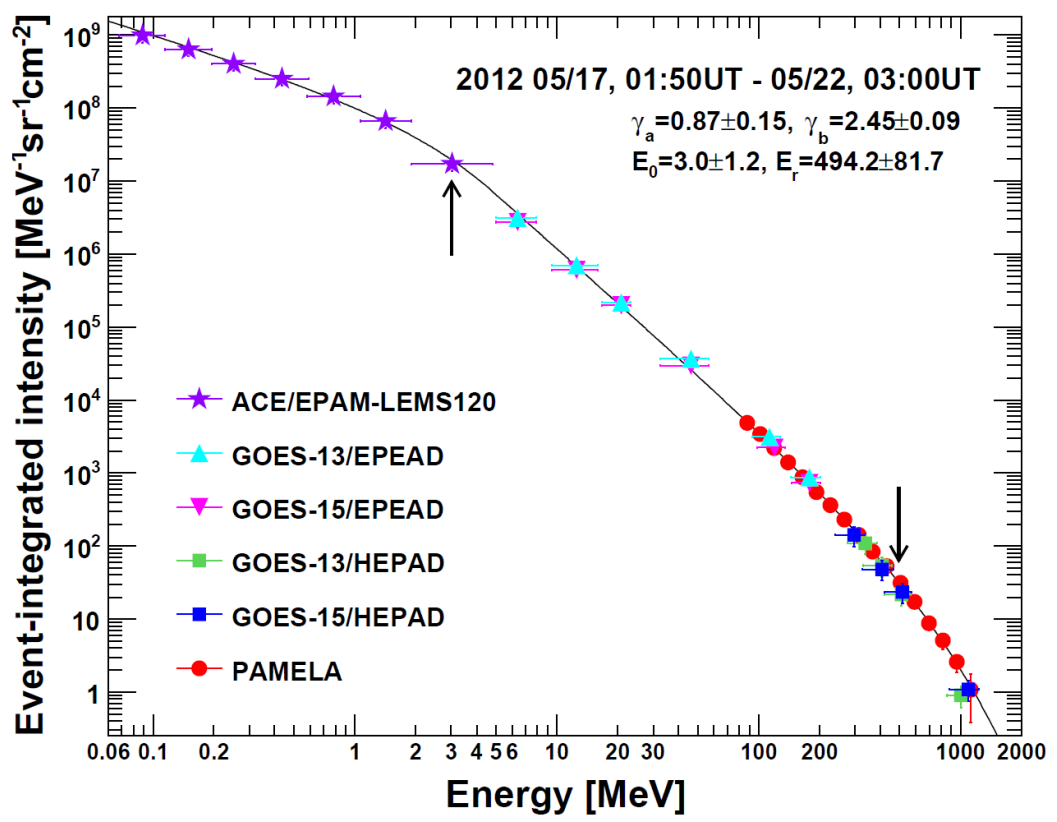

Figure 1: 2012 May 17 SEP event-integrated intensity spectra measured by near-Earth spacecraft. The solid line is a fit performed by using a combined functional form, with corresponding parameters reported along with the integration intervals. The upward and downward pointing arrows mark the break and the rollover energies, respectively [1]. 

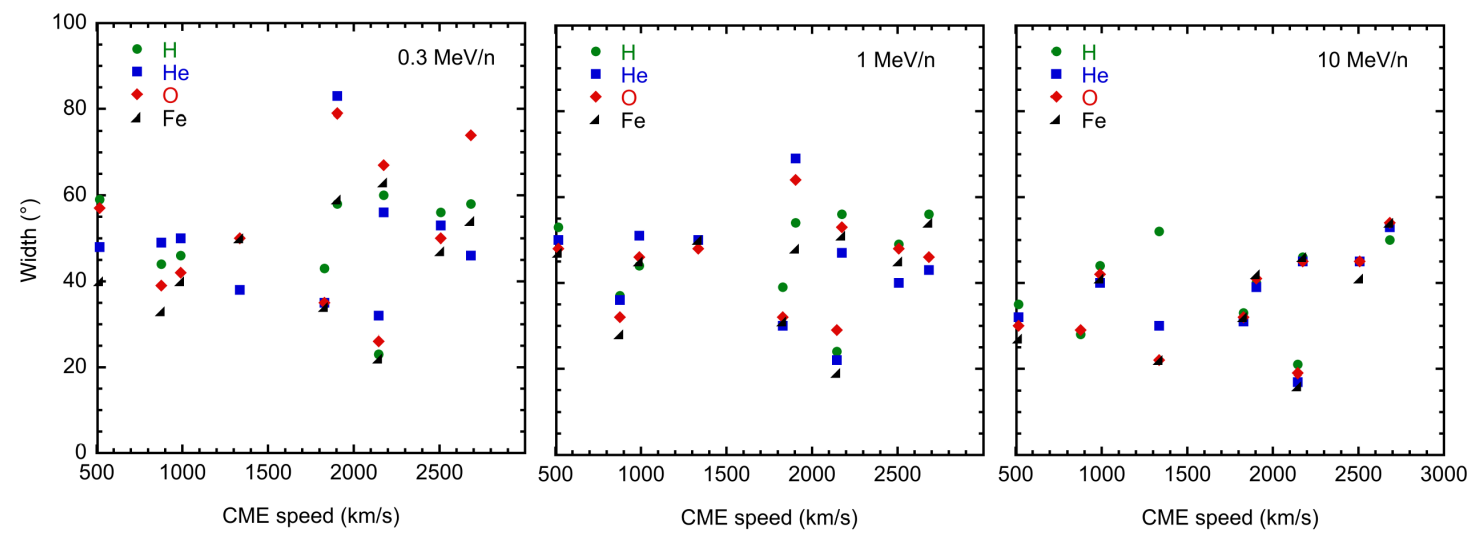

Figure 2: Plots of the calculated longitudinal widths of three-spacecraft events versus CME speed. The left column plots are widths of $0.3 \mathrm{MeV} / \mathrm{n}$ ions, the center column is for $1 \mathrm{MeV} / \mathrm{n}$ ions and the right column is $10 \mathrm{MeV} / \mathrm{n}$. In each plot the colored symbols identify the species, H, He, O and Fe [3].
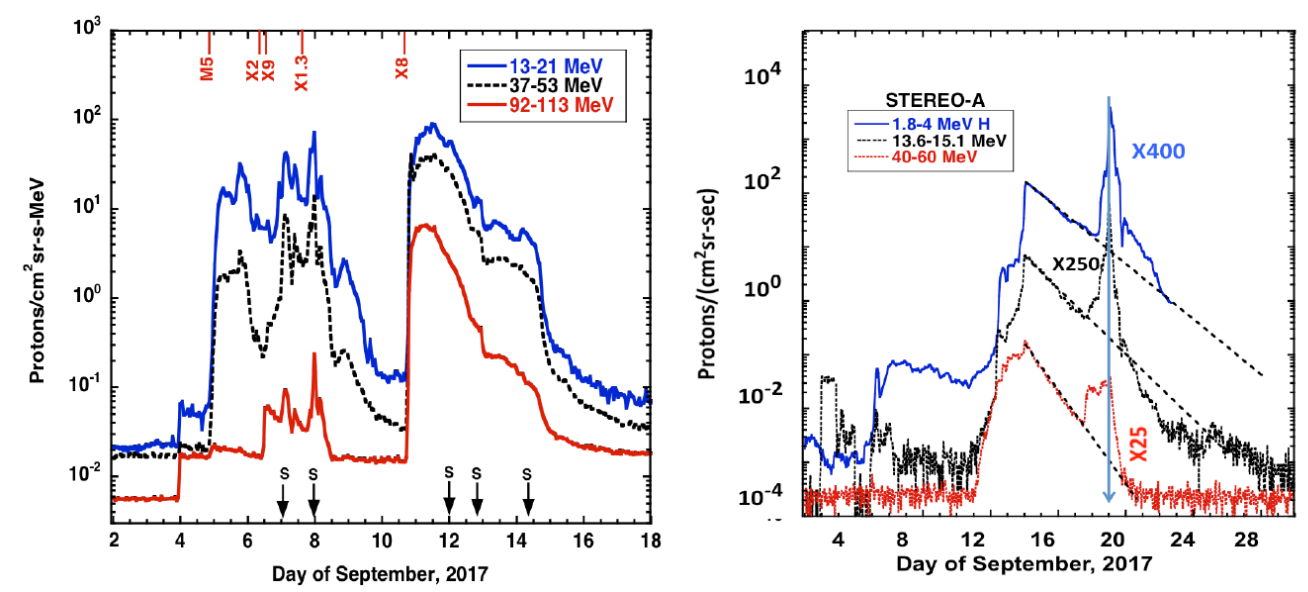

Figure 3: The time history of the September 2017 SEP activity as seen by GOES-13 (left) and STEREO A (right) [4].

over which measurements have been made routinely for ions is approximately $0.1-100 \mathrm{MeV} / \mathrm{nuc}$. Information on higher energy ions has traditionally be obtained indirectly through data from neutron monitors on Earth's surface, during the relatively rare Ground Level Enhancements (GLEs).

In this ICRC new results have been presented on the detection of $\sim 100 \mathrm{MeV}-1 \mathrm{GeV}$ protons from solar events, from data from the PAMELA spacecraft. Bruno et al [1] have carried out an analysis of SEP spectra for 26 major SEP events over a broad energy range, that includes the PAMELA energy range, with an example shown in Figure 1. The spectrum shows the presence of two features: a break at around $3 \mathrm{MeV}$ and a rollover around $500 \mathrm{MeV}$, with the latter being detectable thanks to the higher energy resolution of PAMELA, compared to previous instruments. Bruno et al [1] interpreted the rollover feature as being due to the limited spatial extension and limited lifetime of the CME-driven shock as an accelerator. The reason for the presence of the low energy break, which had previously been ascribed to the limited acceleration capability of the CME shock, remains unclear, with a possible explanation being interplanetary transport effects. It 


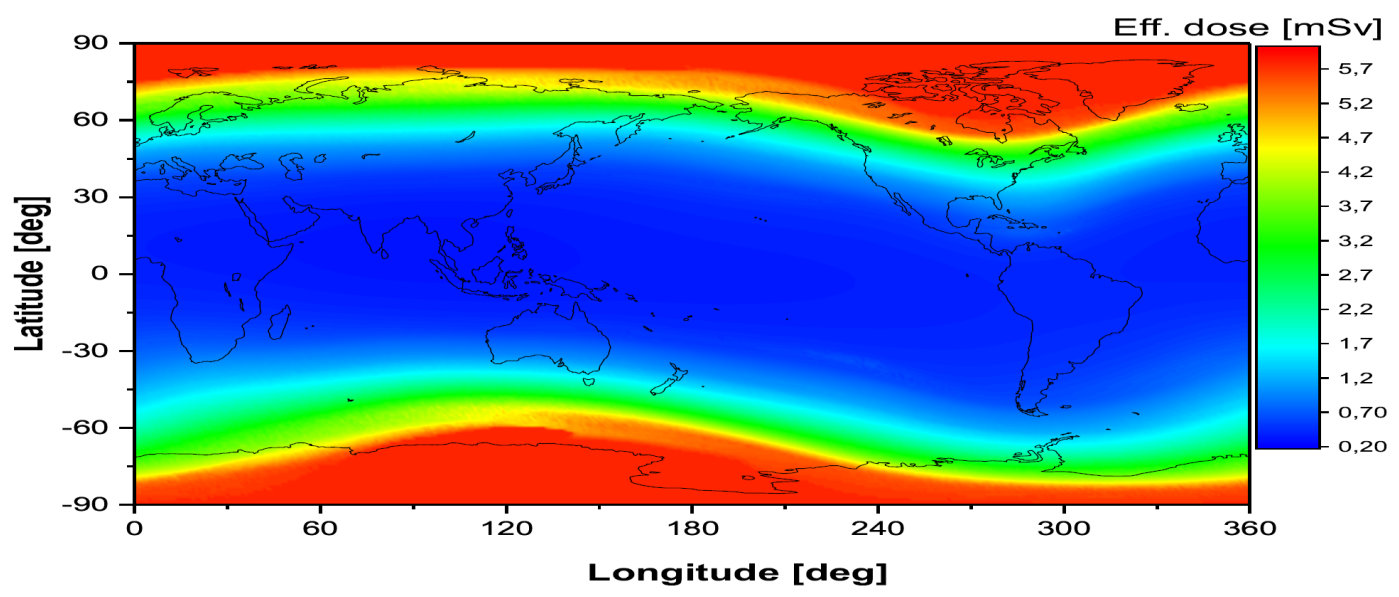

Figure 4: Effective radiation dose integrated over the first 3 hours of GLE 5, at an altitude of $35 \mathrm{kft}$.

was pointed out that there is no qualitative difference between the spectra of GLEs and those of non-GLE SEP events. It was noted during the discussion of this work that the shape of the spectrum resembles that expected from second order Fermi acceleration [2], while theory for diffusive shock acceleration predicts a spectrum that becomes harder at higher energies.

Cohen et al [3] carried out a study to evaluate the influence of CME properties on the spatial width in heliographic longitude of SEP events. Data from STEREO A, STEREO B and ACE were analysed for $\mathrm{H}, \mathrm{He}, \mathrm{O}$ and $\mathrm{Fe}$ in the $0.3-10 \mathrm{MeV} /$ nuc energy range. By plotting the peak intensities for each 3-spacecraft event analysed versus longitudinal separation between spacecraft footpoint and flare location, and fitting a gaussian, the width of each SEP event was determined. Figure 2 shows the event width for the ionic species considered, versus the CME speed, as obtained from coronagraph observations. It was found that the correlation between event width and CME speed is either very weak or non-existent. Other properties including CME mass, kinetic energy and acceleration also do not correlate with the observed width.

Mewaldt et al [4] analysed the September 2017 SEP events, using data from near-Earth spacecraft and STEREO A. Figure 3 shows time intensity profiles of energetic protons at the two spacecraft as well as indicating the intense flaring activity during this period. The detected particle enhancements are quite different at STEREO A compared to near-Earth, with the two locations separated by $\sim 130$ degrees. The data shows the complexity of interpreting data from widely separated spacecraft during times of high solar activity. The extreme 'spike-like' nature of the September 2017 Energetic Storm Particle (ESP) event at STEREO-A was discussed by Mewaldt et al [4], with the ESP enhancement being by a factor of 400. A strong enhancement was seen for a variety of species including electrons, $\mathrm{C}, \mathrm{Si}$ and $\mathrm{Mg}$. This shows that the September 18th CME shock was a very efficient accelerator in spite of its $1380 \mathrm{~km} / \mathrm{s}$ launch speed. Possible interpretations that may explain why the ESP event was so intense at STEREO A include the presence of a large seed population, the shock being quasi-perpendicular, and the magnetic field being particularly strong (20 nT).

SEPs are an important radiation hazard for humans in space and for airline passegers and crews. Mishev et al [5] presented a methodology by which, starting from neutron monitor data, 


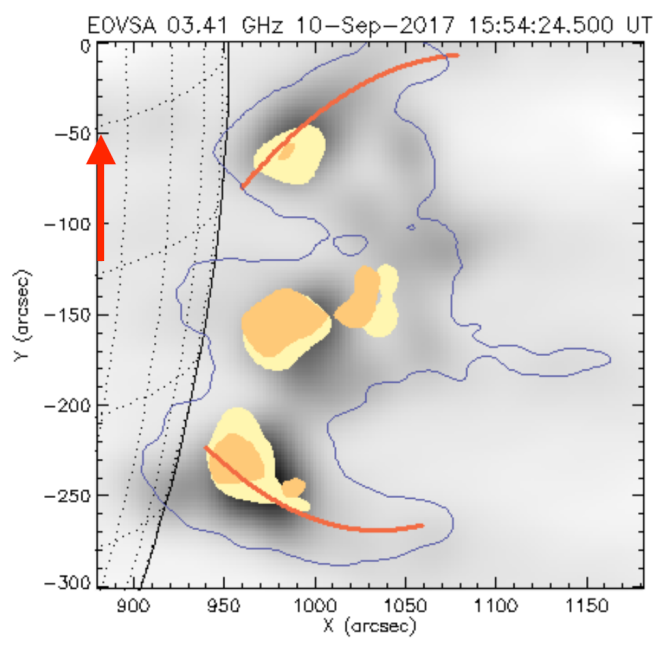

Figure 5: EOVSA 3.4 GHz image for the 2017 September 10th event. The legs of large energized coronal loops are indicated in red, just over the west limb [6].

the effective dose can be derived at a variety of altitudes. The calculation takes into account the dynamical variation of the SEP spectra and uses a fit with either a modified power-law rigidity spectrum or an exponential spectrum. Figure 4 shows the effective dose at $35 \mathrm{kft}$, corresponding to aviation altitudes, over the first 3 hours of GLE 5, one of the early GLE events.

\subsection{Long duration $\gamma$-ray flares}

The Fermi spacecraft, launched in 2008, has detected tens of long duration $\gamma$-ray emission events, lasting for several hours and continuing well after other electromagnetic emission has ceased. Previously thought to be rare, these types of events have been shown to be relatively common. Gamma-ray radiation is though to be generated by pion decay triggered by precipitation onto the solar photosphere of high energy ions, with the majority of the emission being caused by protons of energy $>300 \mathrm{MeV}$.

The mechanism by which precipitation of relativistic ions onto the photosphere can take place continuously over timescales of several hours is unclear. One possible explanation is continuous acceleration by a CME-driven shock as it propagates outwards, followed by back-precipitation of particles from the accelerating shock to 1 solar radius. Ryan et al [6] discussed a second possible explanation involving trapping and acceleration (by 2 nd order Fermi processes) in large coronal loops. For the 2017 September 10th event, Figure 5 shows radio imaging data at $3.4 \mathrm{GHz}$, displaying enhanced emission over the legs of a large coronal loop. They proposed an interpretation according to which relativistic protons were trapped inside the loop by strong diffusion as well as accelerated and suggested that the same particles were responsible for the long duration $\gamma$-ray emission detected for this event [6].

de Nolfo et al [7] presented a comparison between numbers of energetic protons at the Sun, as inferred from long duration $\gamma$-ray flare data, and numbers at $1 \mathrm{AU}$, obtained from an analysis of PAMELA and STEREO A and B data, combined with transport simulations. Figure 6 displays proton number at the Sun versus proton number at $1 \mathrm{AU}$, for 14 Fermi/LAT events, showing these 


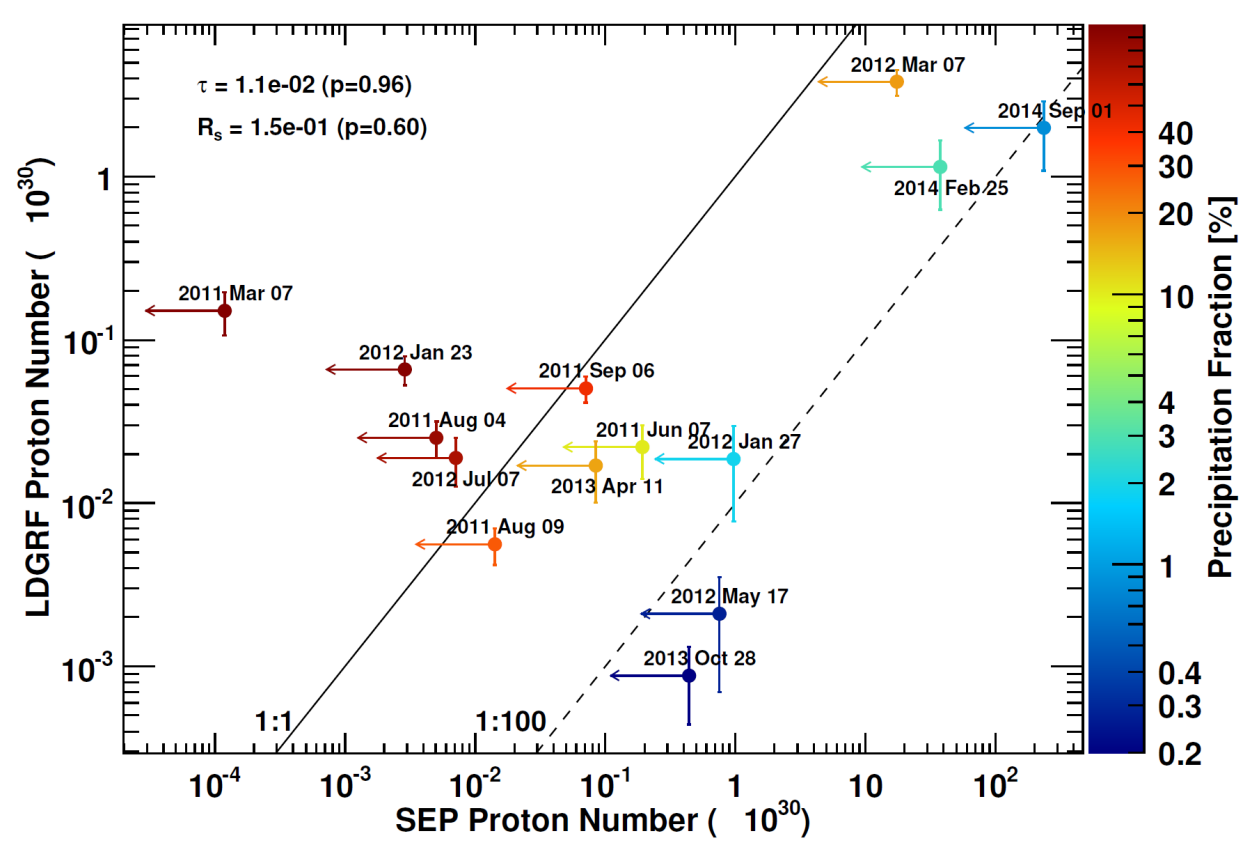

Figure 6: Number of protons at the Sun deduced from Fermi/LAT data compared with number of protons in interplanetary space determined from PAMELA and STEREO-A/B data at $1 \mathrm{AU}$. The color code shows the precipitation fraction. The solid and the dashed lines mark the 1:1 and the 1:100 correspondences, respectively [7].

numbers are not correlated. In many events, the numbers at the Sun and at $1 \mathrm{AU}$ are similar (see 1:1 line (solid line)). de Nolfo et al [7] pointed out that this observation poses a problem to the CME acceleration and back-precipitation model. In fact, the converging magnetic fields as one moves towards the Sun cause a strong mirror effect, so that the expectation is that only a small fraction of the particles accelerated by a CME driven shock would be able to propagate back down to the photosphere.

\subsection{Anomalous cosmic rays}

A second important species of energetic particles within the heliosphere are Anomalous Cosmic Rays (ACRs). These ions, in the energy range $\sim 1-30 \mathrm{MeV} /$ nuc, were previously thought to be accelerated at the heliospheric termination shock (TS). However, observations from the Voyager spacecraft did not show a large enhancement in the intensity of ACRs near the TS: this has led to a new hypothesis that the main site of acceleration at the TS may be its flanks and that the accelerated ACRs propagate along the shock towards its nose.

Cummings et al [9] presented anisotropy measurements at Voyager 2 for $0.5-35 \mathrm{MeV}$ protons, as shown in Figure 7. Here $\mathrm{T}$ and $\mathrm{N}$ components of the diffusive anisotropy vectors were obtained after subtraction of the Compton-Getting anisotropy. They show an ACR diffusive flow equatorward and towards the nose of termination shock, from the flank or tail. These results were interpreted to support models that have the TS flank or tail as main acceleration site for ACRs [9].

ACR O, $\mathrm{N}$ and Ne during the last two solar minima were analysed by Leske et al [8]. They considered ACR intensities during times of high solar wind speeds $(>550 \mathrm{~km} / \mathrm{s})$ and low solar 


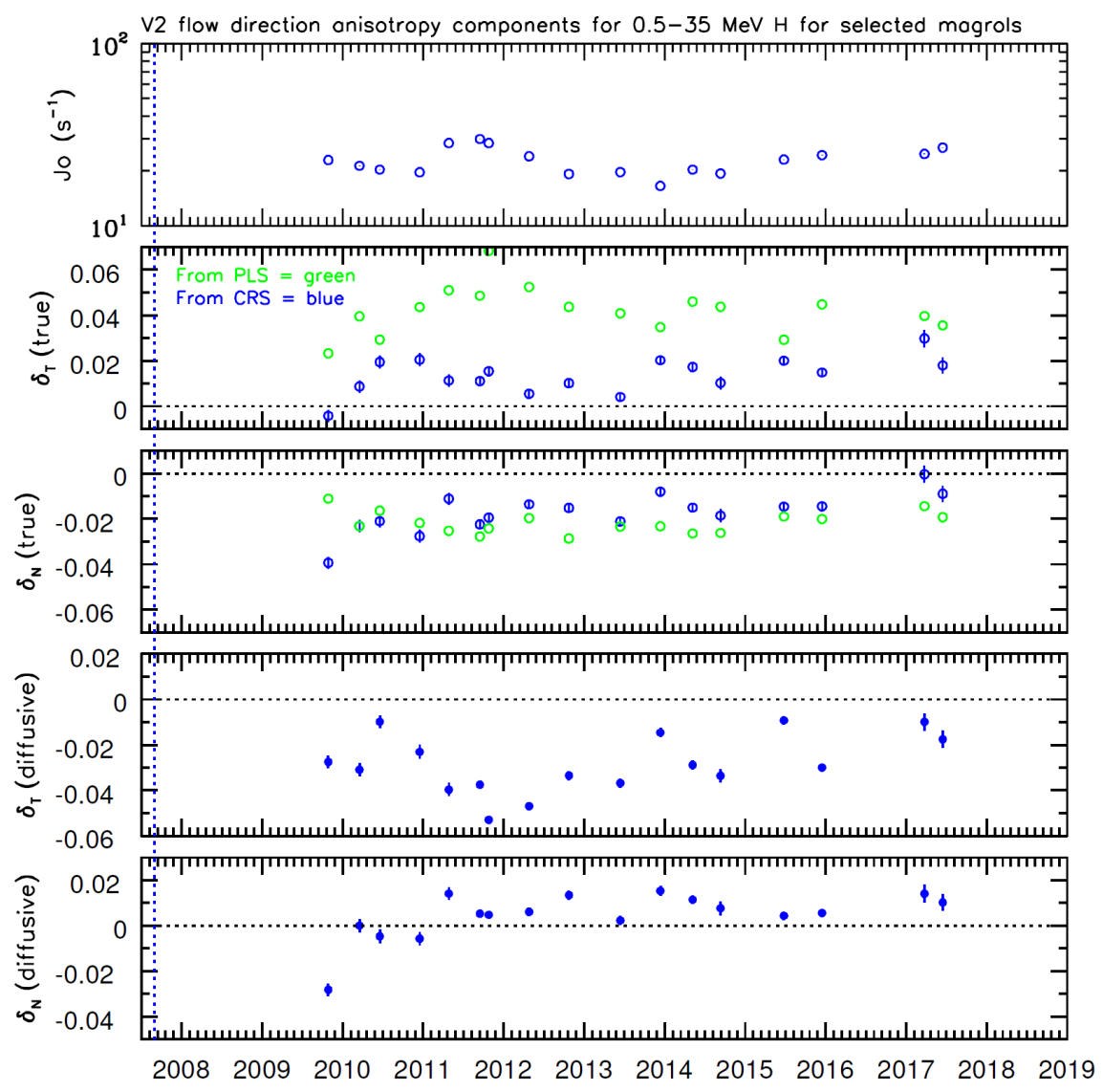

Figure 7: ACR anisotropies from Voyager 2 in the inner heliosheath. The panels show the omindirectional flux $J_{0}$ and T and N components of the anisotropies (in the RTN coordinate system) - see details in [9]. The Compton-Getting components (labeled "From PLS = green") are shown and are based on the corrected solar wind speeds and the energy spectra. The bottom two panels give $\mathrm{T}$ and $\mathrm{N}$ components of the diffusive anisotropy vectors [9].

wind speeds $(<350 \mathrm{~km} / \mathrm{s})$. As shown in Figure 8, they found that during high speed times the ACR intensities are systematically lower than during slow speed times, an effect that is stronger at the low end of the ACR energy range. The trend with solar wind speed is observed for both a time range during the solar cycle 23 minimum and one during the cycle 24 minimum.

Kota et al [10] discussed recent simulations of ACR propagation within a hoop model of the heliospheric magnetic field that included a Heliospheric Current Sheet (HCS). The results of the simulations are displayed in Figure 9, where intensities of ACRs are shown for $A<0$ and $A>0$ polarity configurations. The results reproduce the well known polarity dependence in the meridional distribution of ACRs at solar minima.

\section{How is propagation processing particle fluxes, spectra and other observables?}

Galactic Cosmic Rays (GCRs), originating outside the heliosphere and travelling within it to reach near-Earth space, are routinely detected by $1 \mathrm{AU}$ spacecraft, displaying the well known solar 


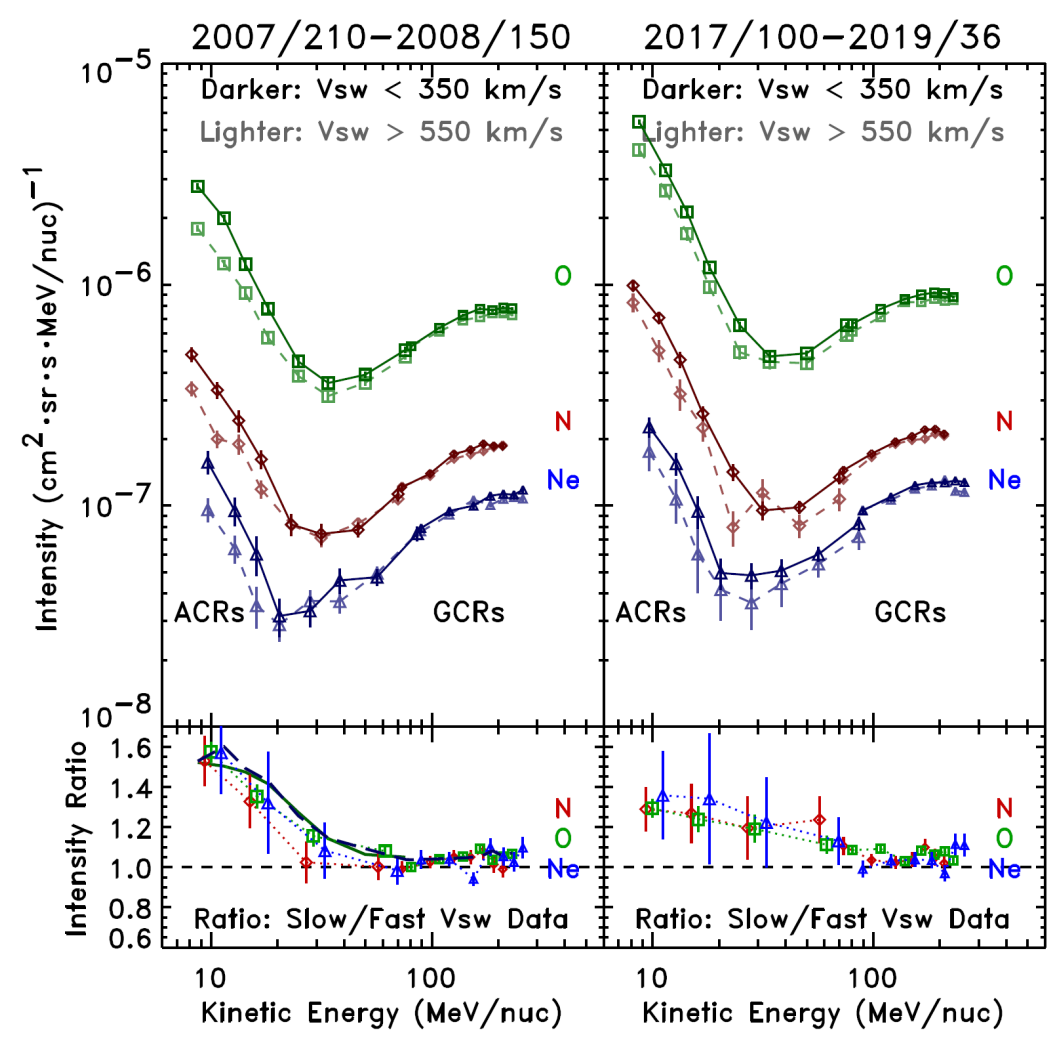

Figure 8: Energy spectra (top panels) from ACE/SIS and ACE/CRIS for O, $\mathrm{N}$ and Ne during the previous solar minimum period (left) and present solar minimum (right). For each element and time interval two spectra are shown, one for periods when the solar wind speed was $>550 \mathrm{~km} / \mathrm{s}$ (lighter dashed curves) and one when the speed was $<350 \mathrm{~km} / \mathrm{s}$ (darker solid curves). Ratios of the spectra at the two solar wind speeds are plotted in the bottom panels [8].

modulation patterns. A number of observations of GCRs at 1 AU have been presented at this ICRC, and they are reviewed within the Cosmic Ray Direct rapporteur paper [11].

Here we focus on modelling results aimed at understanding the propagation of GCRs within the heliosphere and interaction with the solar wind. The main developments presented at this ICRC have involved new techniques to constrain modulation model parameters, with better data for this purpose being now available.

\subsection{GCR modulation models}

Corti et al [13] and Bischhoff et al [12] presented modelling work using a 3D steady state modulation model based on the Parker equation, including solar wind convection, drift, diffusion and energy losses. They used new datasets to constrain modulation model parameters such as drift and diffusion parameters.

Bischhoff et al [12] tuned the modulation model using proton and electron data at Voyager 1 and PAMELA. They also used GALPROP to derive Local Interstellar Spectra for a variety of species. The model thus tuned was then used to predict eg positron and boron spectra, which were found to agree well with measurements from PAMELA. 

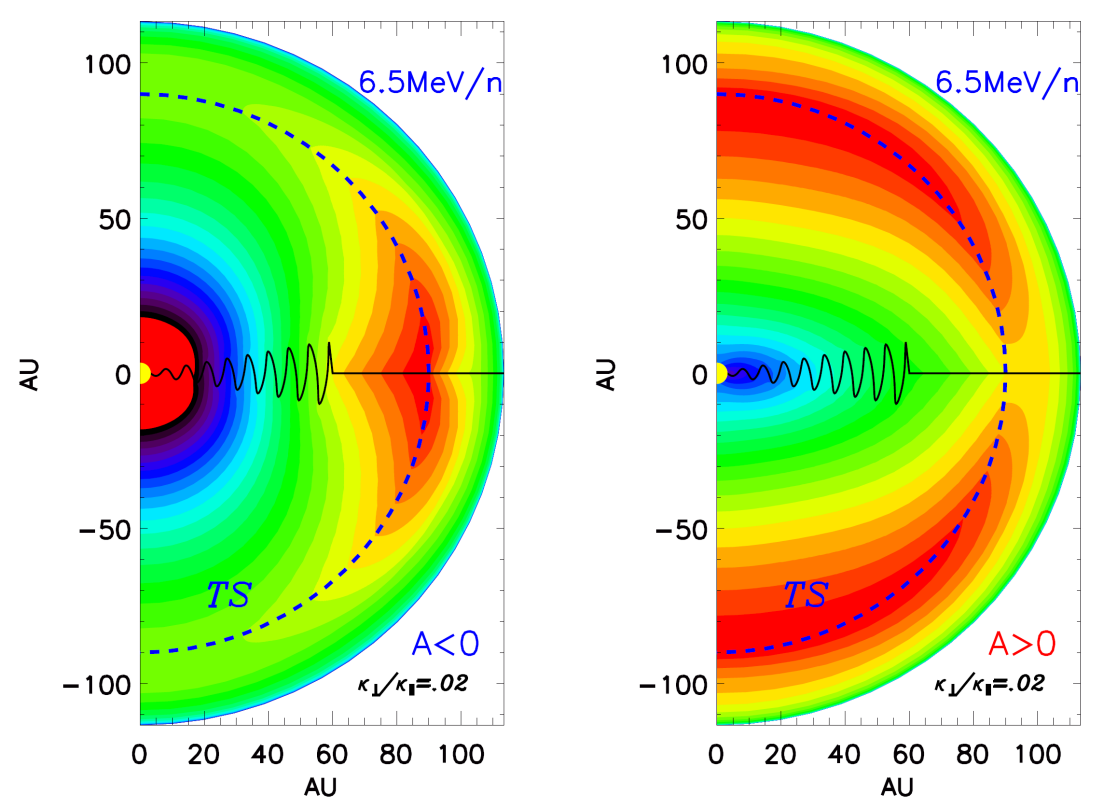

Figure 9: Contour plots of ACR intensity in a kinematical hoop model during two consecutive solar minima. The black line indicate the HCS that is carried out radially by the solar wind [10].

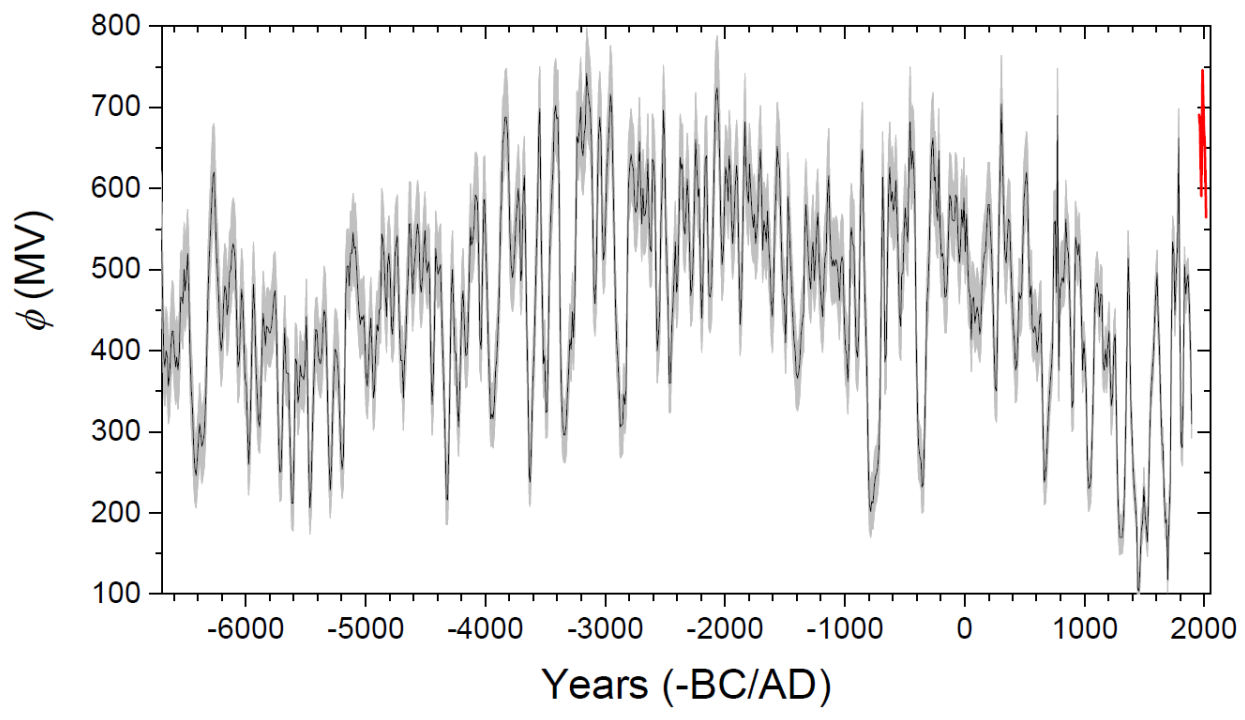

Figure 10: Multi-proxy reconstruction, based on a Bayesian approach, of the decadally averaged cosmic-ray modulation potential for the mean (black curve) and $1 \sigma$ confidence interval (grey shading) for the period $6700 \mathrm{BC}-1900 \mathrm{AD}$. The red curve depicts the decadally averaged modulation potential for the instrumental era, since 1951 [15]. 

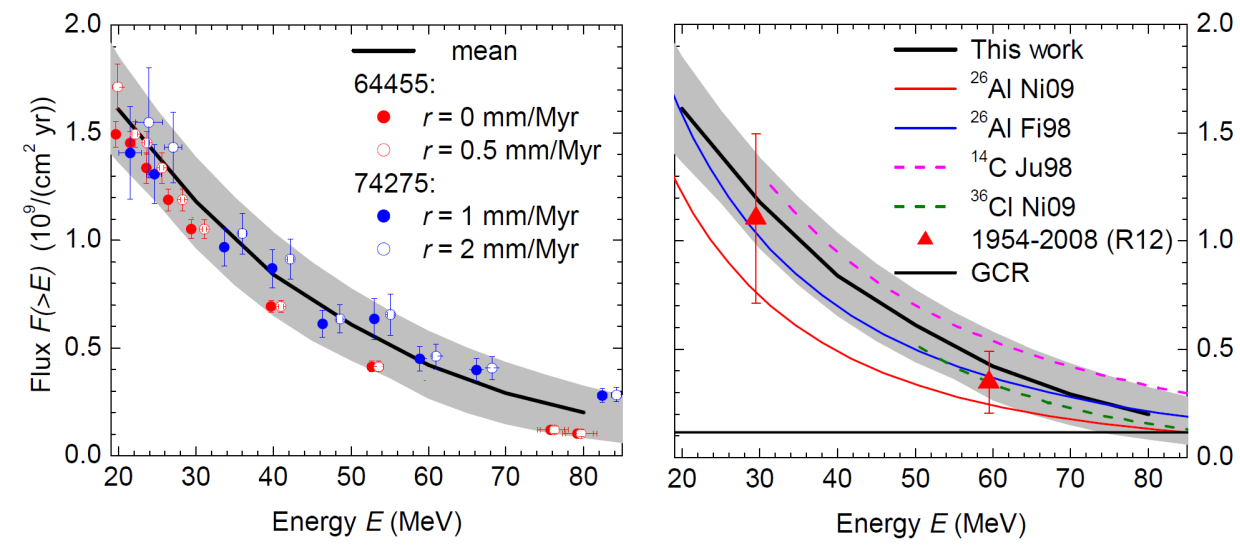

Figure 11: SEP integral omnidirectional flux $F(>E)$ versus $E$. Left panel: reconstruction of the mean spectrum on the Myr time scale from ${ }^{26} \mathrm{Al}$. The black line is the mean reconstruction from two lunar samples with the grey area indicating full-range uncertainties. The circles represent individual reconstructions from those samples for different erosion rates, as specified in the legend. Right panel: The black line is the same as in the left panel, with the other curves having been obtained in previous work. The triangles indicate the results for the modern era [16].

Corti et al [13] tuned their modulation model to fit AMS 1.5 GV proton flux data between mid 2011 and early 2017. For example, the model includes a parametrised rigidity dependence of diffusion, with parameter values that can be constrained by the observations. They then used the model, tuned with proton data, to make a prediction for He fluxes, and showed that the resulting proton to helium ratio at energies in the range $\sim 2-3 \mathrm{GV}$ decreases in time, in agreement with observations. The decrease of $\mathrm{p} / \mathrm{He}$ ratio was interpreted as due to the $A / Z$ dependence of diffusion, where $A$ is the ionic mass number and $Z$ the ionic charge number [13].

\subsection{Solar energetic particles}

Work on the transport of relativistic solar protons was presented: using test particle simulations that include the effects of the polarity of the interplanetary magnetic field and of the Heliospheric Current Sheet (HCS), the 1 AU spatial distributions of relativistic protons was obtained [14]. The results show that transport within the heliosphere at these energies is three-dimensional, with the HCS facilitating particle propagation across longitude and producing patterns of $1 \mathrm{AU}$ crossings that are different for the $A>0$ and $A<0$ configurations.

\section{How have conditions in the heliosphere evolved over time?}

It is possible to study conditions in the heliosphere over thousands of years and even further back. The majority of work in this area is based on analysis of cosmogenic nucleids produced by the interaction of high energy cosmic rays with a variety of atoms.

One important application is the study of how cosmic ray intensities have changed over timescales of thousands of years, allowing scientists to study how solar modulation evolved over such timescales. Usoskin et al [15] presented a new methodology for deriving the cosmic ray modulation potential $\Phi$ using a multi-proxi Bayesian approach. The analysis used multiple ${ }^{14} \mathrm{C}$ and ${ }^{10} \mathrm{Be}$ datasets and 


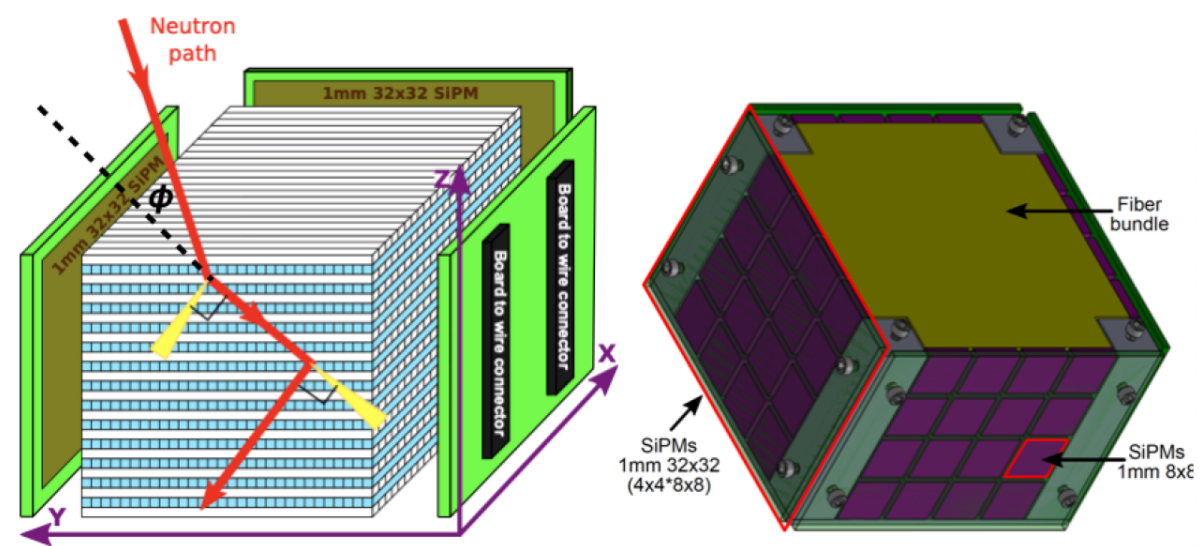

$E_{n o}=E_{p 1}+E_{n s} ; \sin ^{2} \phi=E_{p 1} / E_{n o}$

Figure 12: SONTRAC neutron instrument consisting of orthogonally stacked plastic scintillators readout by arrays of SiPMs. A measurement of two recoil tracks from fast neutron interactions determines the incident neutron energy and direction [17].

obtained $\Phi$ at each time by combining them within an iterative procedure that finds the most probable value. The derived time evolution of the cosmic ray modulation potential is shown in Figure 10. The grand minima observed in this time series agree with previous estimates but the overall behaviour is flatter and, importantly, the uncertainties can be estimated.

Cosmogenic isotopes can also be used to obtain information on the spectra of SEP events over millions of years. Poluianov et al [16] analysed the depth distribution of ${ }^{26} \mathrm{Al}$ in Apollo mission lunar samples, using a new methodology, obtaining the results shown in Figure 11. They found that the SEP spectra on Myr scales are comparable with those of recent decades.

\section{The future: New missions and instrumentation}

This ICRC also included discussion of newly launched or upcoming missions, and of new of proposed instrumentation.

The Parker Solar Probe mission, launched in August 2018, will orbit the Sun over the next $\sim 6$ years, with 24 perihelia taking it down to a radial distance of 9.9 solar radii. The four main instruments were described: the Fields Experiment (FIELDS), detecting electric and magnetic fields and waves; the Integrated Science Investigation of the sun (ISIS), measuring energetic electrons and heavy ions; the Wide-field Imager for Solar PRobe (WISPR), a remote sensing imager of the corona and inner heliosphere; and Solar Wind Electrons Alphas and Protons (SWEAP), detecting solar wind elecrtrons and ions. Preliminary results were presented from several of these investigations.

A report was also given on the Interstellar Mapping and Acceleration Probe (IMAP) mission, planned for launch in 2024. It will include instrumentation to measure Energetic Neutral Atoms with a much higher sensitivity than the earlier IBEX mission, and it will also detect suprathermal and energetic particles at high cadence. 


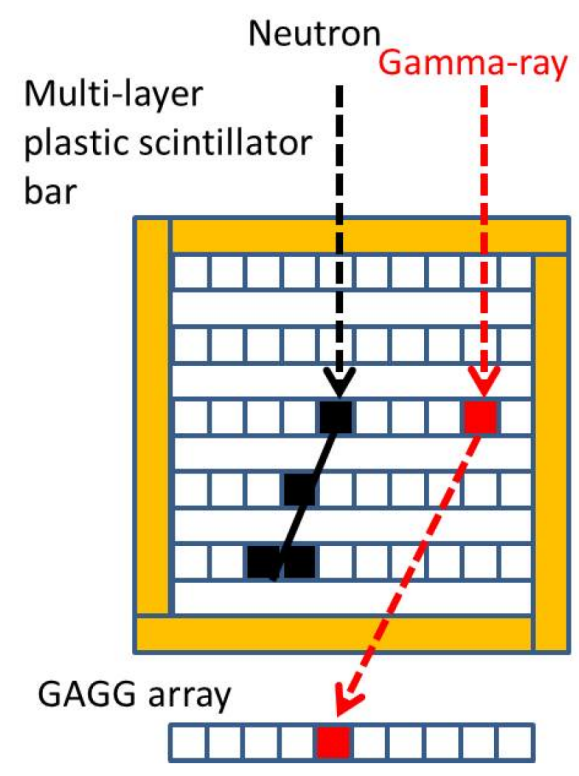

Figure 13: Schematic view of a proposed solar neutron and gamma-ray detector. The detector consists of multi-layered plastic scintillator bars, GAGG(Ce) inorganic scintillator array, and surrounding anticoincidence shield to reject charged particles (shown in orange). Neutrons are detected via elastic scattering with atoms in the plastic scntillator bars, while $\gamma$-rays are detected via Compton scattering. [18].

Among new instrumentation being developed, de Nolfo et al [17] presented the SOlar Neutron TRACking (SONTRAC) concept, as shown in Figure 12. Here scintillating-fiber bundles are used to overcome difficulties in measuring high-energy (>50 MeV) neutrons.

A solar neutron and $\gamma$-ray detector for a $3 \mathrm{U}$ cubesat was presented by Yamaoka et al [18]. It consists of multi-layered plastic scintillator bars and a GAGG scintillator array, as shown schematically in Figure 13.

The recently balloon-borne magnetic spectrometer AESOP-Lite was discussed by Mangeard et al [19], aiming to measure electrons and positrons in the range 20-300 MeV. A schematic diagram of the instrument is shown in Figure 14. Its first flight took place in May 2018 [19]. The data provide an important $1 \mathrm{AU}$ baseline for Voyager measurements. First results on the spectrum of electrons and positrons were presented by Mechbal et al [20], investigating the negative slope below $100 \mathrm{MeV}$.

Among new techniques, Banglieng [21] discussed a method for obtaining spectral information from Neutron Monitor (NM) data from a single station, based on recording neutron time-delay histograms and extracting the 'leader fraction' (inverse multiplicity). Time delays smaller than $4 \mathrm{~ms}$ were taken to represent correlated arrival of neutrons.

A number of reports on neutron monitor improvements and experiments were presented, as detailed in these proceedings. Blanco et al [22] discussed the results of a latitudinal survey on board a research vessel by the Antarctic Cosmic Ray Observatory (ORCA), an instrument comprising a neutron detector, a muon telescope and a mini neutron monitor. 


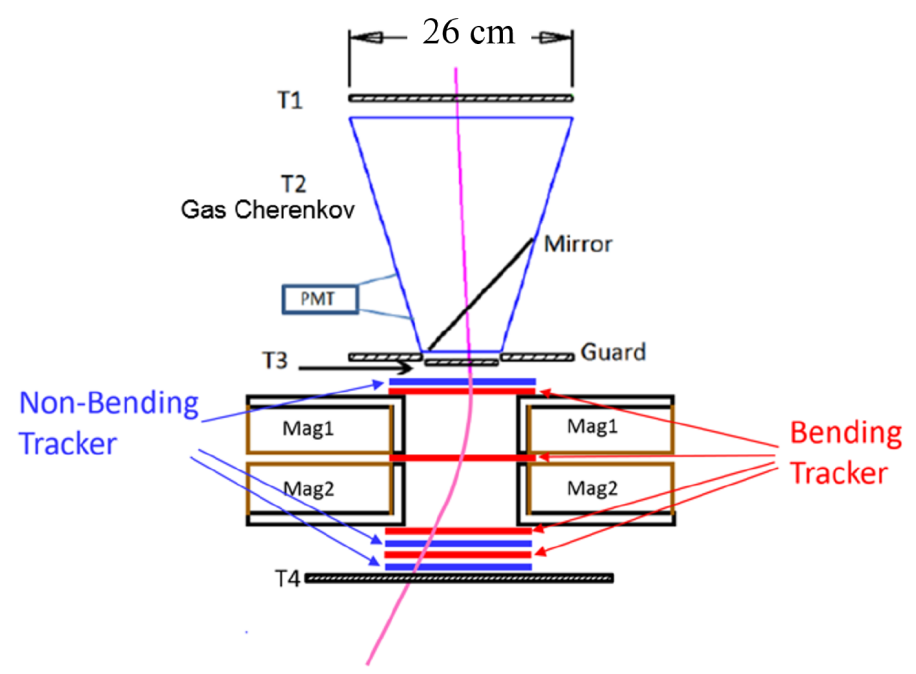

Figure 14: Diagram of the AESOP-Lite instrument. The spectrometer consists of 7 planes of silicon strip detectors. [19].

\section{Conclusion}

The presentations at the 36th ICRC in the area of Solar and Heliospheric Physics contributed new understanding in a number of important topics. Among the highlights were new data on high energy SEPs, and on long duration solar $\gamma$-ray emission, key to unsolved questions on solar particle acceleration.

Models of GCR modulation of increased sophistication were presented and new datasets used to constrain them.

There has been significant progress on understanding past behaviour of solar modulation (over times scales of thousands of years) and SEP flux spectra (over time scales of millions of years).

Finally, recently launched and upcoming missions such as Parker Solar Probe and IMAP will provide key new solar and heliospheric data in future years.

\section{References}

[1] A. Bruno, et al, in Proceedings of 36th ICRC, PoS (ICRC2 019) 1061 (2019).

[2] R. Cowsik, and S. Sarkar, MNRAS, 207, 745 (1984)

[3] C.M.S. Cohen, et al, in Proceedings of 36th ICRC, POS ( ICRC2019) 1066 (2019).

[4] R.A. Mewaldt, et al, in Proceedings of 36th ICRC, POS (ICRC2019) 1120 (2019).

[5] A. Mishev, et al, in Proceedings of 36th ICRC, PoS ( ICRC2019) 1123 (2019).

[6] J.M. Ryan, et al, in Proceedings of 36th ICRC, PoS ( ICRC2019) 1144 (2019).

[7] G.A. de Nolfo, et al, in Proceedings of 36th ICRC, POS ( ICRC2019) 1073 (2019).

[8] R.A. Leske, et al, in Proceedings of 36th ICRC, POS ( ICRC2 019) 1105 (2019).

[9] A.C. Cummings, et al, in Proceedings of 36th ICRC, PoS ( ICRC2019) 1071 (2019). 
[10] J. Kota, et al, in Proceedings of 36th ICRC, POS ( ICRC2019) 1096 (2019).

[11] R. Sparvoli, Cosmic Ray Direct Rapporteur contribution, in Proceedings of 36th ICRC, POS (ICRC2019) 032 (2019).

[12] D. Bisschoff, M. S. Potgieter, \& O. P. M. Aslam, ApJ, 878, 59 (2019)

[13] C. Corti, M. S. Potgieter, V. Bindi, et al, ApJ, 871, 253 (2019)

[14] M. Battarbee, S. Dalla, \& M. S. Marsh, ApJ, 854, 23 (2018)

[15] I. Usoskin, et al, in Proceedings of 36th ICRC, POS ( ICRC2019) 1164 (2019).

[16] S.V. Poluianov, et al, in Proceedings of 36th ICRC, POS ( ICRC2019) 1139 (2019).

[17] G.A. de Nolfo, et al, in Proceedings of 36th ICRC, POS ( ICRC2019) 1074 (2019).

[18] K. Yamaoka, et al, in Proceedings of 36th ICRC, POS ( ICRC2019) 1173 (2019).

[19] P.S. Mangeard, et al, in Proceedings of 36th ICRC, POS ( ICRC2019) 1116 (2019).

[20] S. Mechbal, et al, in Proceedings of 36th ICRC, POS ( ICRC2019) 1119 (2019).

[21] C. Banglieng, et al, in Proceedings of 36th ICRC, POS ( ICRC2019) 1055 (2019).

[22] J.J. Blanco, et al, in Proceedings of 36th ICRC, PoS ( ICRC2019) 1059 (2019). 